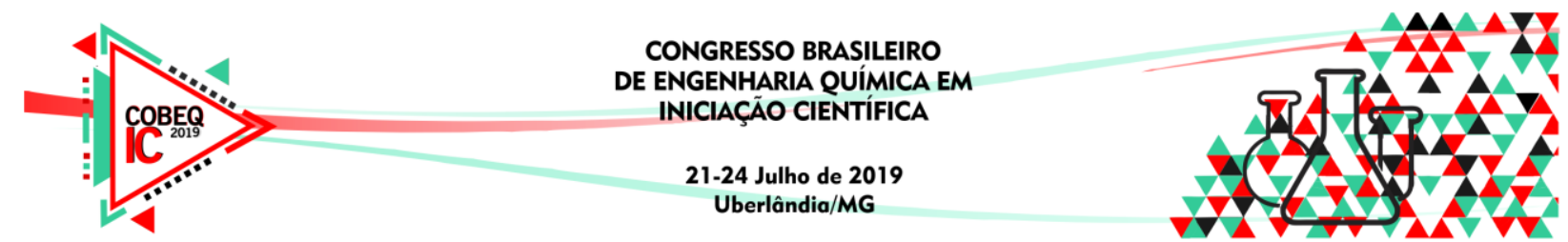

\title{
IMPACTO DA SECAGEM POR MICRO-ONDAS DE FORMA CONTÍNUA E INTERMITENTE NOS COMPOSTOS BIOATIVOS DA MICROALGA Spirulina platensis
}

\author{
A. L. V. FUGEIRO ${ }^{1}$, I. S. GRATON ${ }^{1}$, N. C. SILVA', C. R. DUARTE e M. A. S. \\ BARROZO' ${ }^{1}$. \\ ${ }^{1}$ Universidade Federal de Uberlândia, Faculdade de Engenharia Química \\ E-mail para contato: masbarrozo@ufu.br
}

\begin{abstract}
RESUMO - A Spirulina platensis é uma microalga com inúmeras propriedades e qualidades nutricionais, que tem vitaminas e antioxidantes que podem ser utilizadas para fins alimentícios, farmacêuticos, entre outros. No entanto, os altos teores de água presentes nesse material dificultam sua utilização, sendo necessário, portanto, um método de secagem para torná-la apta para o uso e armazenamento. Dentre as técnicas existentes, destaca-se a secagem por microondas, reconhecida pelos baixos tempos de processo e eficiência energética. Neste trabalho avaliou-se a secagem da Spirulina em micro-ondas de forma contínua e intermitente, nas potencias de $200 \mathrm{~W}, 280 \mathrm{~W}, 480 \mathrm{~W}, 600 \mathrm{~W}$ e $800 \mathrm{~W}$, e seus efeitos nas concentrações dos compostos bioativos presentes no material (fenólicos, flavonoides, ficocianina e ácido cítrico). Verificou-se que apesar da degradação de parte dos bioativos após a secagem, foi possível obter, em condições específicas, teores mais elevados em potências intermediárias, principalmente se realizada de forma intermitente.
\end{abstract}

\section{INTRODUÇÃO}

A Spirulina platensis é uma microalga fotossintetizante e multicelular que apresenta alto potencial nutricional graças à presença de proteínas e vitaminas em sua composição. Apresenta compostos e pigmentos antioxidantes, como a ficocianina, que permitem sua aplicação pela indústria de alimentos, cosméticos, farmacêutica e de pigmentos além de diversos outros fins comerciais, como, por exemplo, material adsorvente de materiais pesados (DESMORIEUX \& DECAEN, 2005).

Devido aos elevados teores de água presentes na Spirulina in natura, essa microalga apresenta um alto teor de degradação. Ao ser mantida à temperatura ambiente durante um certo período de tempo, a biomassa úmida presente na microalga começa a ser utilizada por bactérias e inicia-se a sua degradação (DOKE JR, 2005). Sendo assim, faz-se necessário um método de preservação para um melhor aproveitamento da microalga. Dentre esses métodos, destaca-se a secagem por micro-ondas, que tem se destacado por ser mais rápida, uniforme e apresentar uma eficiência energética maior se comparada a outros métodos convencionais, além de ser realizada em equipamentos compactos e de fácil manuseio (VENKATESH \& RAGHAVAN, 2004). 


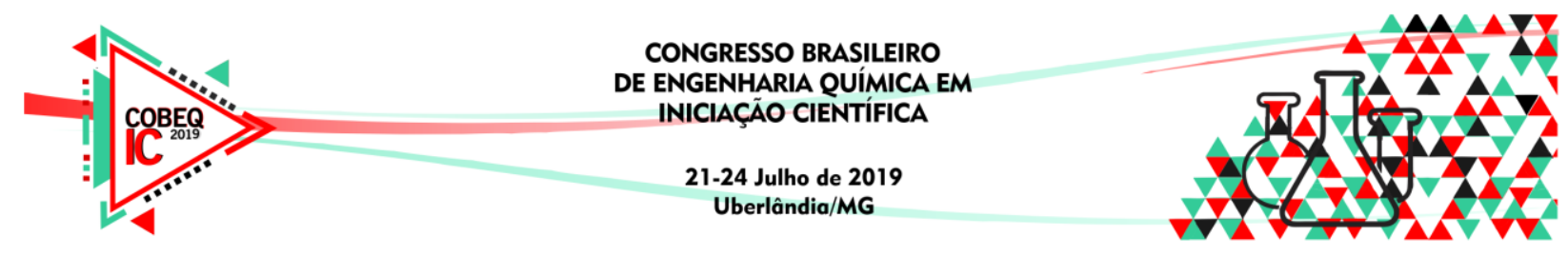

Assim, o objetivo desse trabalho foi realizar a secagem da Spirulina por micro-ondas, submetendo-a a diferentes potências de forma contínua e intermitente, e analisar a variação dos teores de compostos bioativos na microalga após a secagem. Foram avaliados os compostos bioativos fenólicos, flavonoides e ficocianina, além da acidez da amostra.

\section{MATERIAIS E MÉTODOS}

\subsection{Resíduo Utilizado}

A Spirulina platensis utilizada foi fornecida pela Brasil Vital, uma empresa de Anápolis-GO. O material foi previamente filtrado a vácuo e envolto em embalagens de polietileno cobertas de papel alumínio para evitar a sua fotodegradação. Essas embalagens foram levadas ao freezer e congeladas à $-18^{\circ} \mathrm{C}$ até o momento dos experimentos.

\subsection{Aparato Experimental}

Figura 1 - Sistema de Micro-ondas modificado para os experimentos.

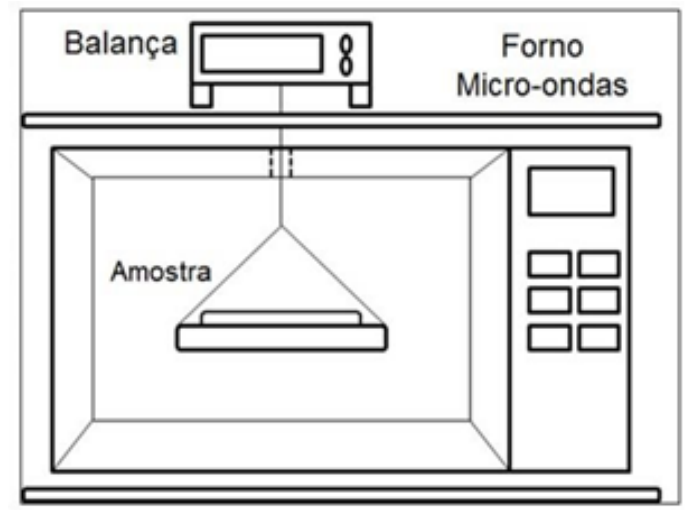

Para realizar a secagem, foi utilizado um micro-ondas doméstico convencional (Panasonic, NN-SF560WRU) de potência nominal $800 \mathrm{~W}$ acoplado a uma balança analítica (Figura 1), de modo que não fosse preciso interromper o experimento para pesagem e coleta de dados. A secagem da Spirulina foi realizada de duas formas. A primeira, de forma contínua, onde manteve-se a mesma potência durante todo o processo em cinco experimentos distintos $(200 \mathrm{~W}, 280 \mathrm{~W}, 480 \mathrm{~W}, 600 \mathrm{~W}$ e $800 \mathrm{~W})$; a segunda, de forma intermitente, na qual variou-se o fornecimento de energia (isto é, a potência) quando o material apresentava $50 \%$ de umidade. Para isso, foram feitos 10 experimentos nos seguintes intervalos de potência: $800 \mathrm{~W}$ a $600 \mathrm{~W}, 800 \mathrm{~W}$ a $480 \mathrm{~W}, 800 \mathrm{~W}$ a $280 \mathrm{~W}, 800 \mathrm{~W}$ a $200 \mathrm{~W}, 600 \mathrm{~W}$ a $480 \mathrm{~W}, 600 \mathrm{~W}$ a $280 \mathrm{~W}, 600 \mathrm{~W}$ a $200 \mathrm{~W}, 400 \mathrm{~W}$ a $280 \mathrm{~W}, 400 \mathrm{~W}$ a $200 \mathrm{~W}$ e $280 \mathrm{~W}$ a $200 \mathrm{~W}$. 


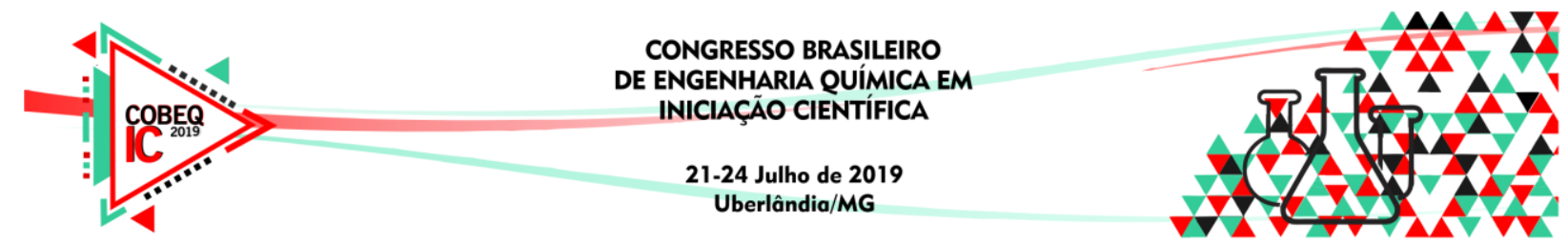

\subsection{Análises Realizadas}

A determinação da umidade das amostras foi feita pelo método da estufa, através de uma estufa de convecção forçada, à $105 \pm 3^{\circ} \mathrm{C}$ durante 24 horas (AOAC, 1995). Já a atividade de água $\left(\mathrm{a}_{\mathrm{w}}\right)$ foi determinada pelo aparelho LabSwift da Novasina, o qual forneceu leituras na faixa de 0,000 a 1,000 com precisão de $\pm 0,001$.

Quanto às análises de compostos bioativos, avaliou-se os compostos fenólicos, flavonoides, acidez e ficocianina. O Teor de Fenólicos Totais (TPC) foi determinado pelo método de Folin- Ciocalteau, utilizando-se ácido gálico $\left(\mathrm{C}_{7} \mathrm{H}_{6} \mathrm{O}_{5}\right)$ como padrão e leitura em espectrofotômetro, à $622 \mathrm{~nm}$ (SINGLETON \& ROSSI, 1965). Os resultados foram expressos em $\mathrm{mg}$ ácido gálico/100 g de amostra seca. O Teor de Flavonoides Totais (TFC) foi determinado através de método colorimétrico também com leitura em espectrofotômetro, à $450 \mathrm{~nm}$ (ZHISHEN ET AL., 1999). A rutina $\left(\mathrm{C}_{27} \mathrm{H}_{30} \mathrm{O}_{16}\right)$ foi utilizada como padrão e os resultados foram expressos em mg de rutina / $100 \mathrm{~g}$ amostra em base seca. A Acidez (ATT), que consiste em quantificar a presença de ácido cítrico nas amostras, foi realizando titulandose as mesmas com $\mathrm{NaOH}$ até a viragem (AOAC, 1995). Os resultados obtidos foram expressos em mg de ácido cítrico / $100 \mathrm{~g}$ amostra em base seca. O Teor de Ficocianina (TF) foi determinado por método adaptado de Costa et al. (2016) pela leitura em espectrofotômetro à 620 e $652 \mathrm{~nm}$. Os resultados foram expressos em $\mathrm{g}$ ficocianina/100 $\mathrm{g}$ em base seca.

\section{RESULTADOS E DISCUSSÕES}

\subsection{Umidade e Atividade de Água}

Analisando a Tabela 1, foi possível observar que essa metodologia apresentou resultados muito positivos, tanto em relação à umidade, que foi reduzida para teores menores que $10 \%$, quanto em relação à atividade de água, indicando que seus teores foram reduzidos com eficiência. Esse fato foi observado em ambas os métodos de secagem: contínuo e intermitente. Além disso a secagem por micro-ondas, quando realizada em altas potências, mostra-se eficiente também em relação ao tempo de processo, sendo capaz de desidratar a amostra em tempos reduzidos. $\mathrm{O}$ mesmo não acontece se a potência for reduzida; isso pode ser explicado pela falta de força motriz térmica para remoção da água das amostras, o que dificulta o processo. 


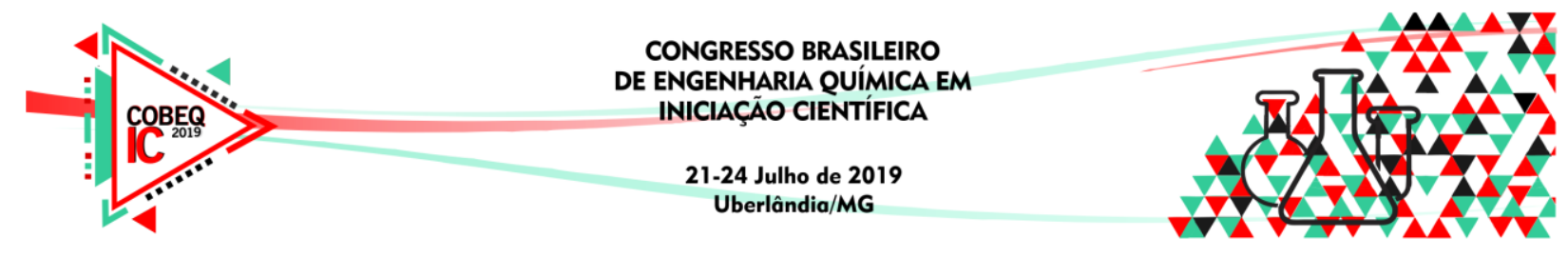

Tabela 1: Resultados da Umidade e Atividade de Água.

\begin{tabular}{|c|c|c|c|c|}
\hline \multirow{3}{*}{ Experimento } & Potência & Umidade (\%) & $\begin{array}{c}\text { Atividade de } \\
\text { Água }\end{array}$ & $\begin{array}{c}\text { Tempo de } \\
\text { Secagem } \\
\text { (min) }\end{array}$ \\
\hline \multirow{4}{*}{$\begin{array}{c}\text { SECAGEM } \\
\text { CONTÍNUA }\end{array}$} & $200 \mathrm{~W}$ & $8,11 \pm 0,03 \%$ & 0,429 & 70 \\
\cline { 2 - 5 } & $280 \mathrm{~W}$ & $6,62 \pm 0,26 \%$ & 0,392 & 33 \\
\cline { 2 - 5 } & $480 \mathrm{~W}$ & $4,46 \pm 0,19 \%$ & 0,347 & 18 \\
\cline { 2 - 5 } & $600 \mathrm{~W}$ & $4,10 \pm 0,08 \%$ & 0,337 & 12 \\
\hline \multirow{7}{*}{ SECAGEM } & $800 \mathrm{~W}$ & $4,48 \pm 0,23 \%$ & 0,342 & 11 \\
\cline { 2 - 5 } & $800 \rightarrow 600 \mathrm{~W}$ & $7,92 \pm 0,08 \%$ & 0,451 & 7,5 \\
\cline { 2 - 5 } & $800 \rightarrow 480 \mathrm{~W}$ & $5,58 \pm 0,06 \%$ & 0,343 & 13,5 \\
\cline { 2 - 5 } & $800 \rightarrow 280 \mathrm{~W}$ & $7,60 \pm 0,18 \%$ & 0,407 & 18,0 \\
\cline { 2 - 5 } & $600 \rightarrow 480 \mathrm{~W}$ & $7,79 \pm 0,09 \%$ & 0,420 & 39,0 \\
\cline { 2 - 5 } & $600 \rightarrow 280 \mathrm{~W}$ & $9,93 \pm 0,09 \%$ & 0,355 & 12,0 \\
\cline { 2 - 5 } & $600 \rightarrow 200 \mathrm{~W}$ & $9,43 \pm 0,12 \%$ & 0,463 & 16,0 \\
\hline & $480 \rightarrow 280 \mathrm{~W}$ & $10,57 \pm 0,17 \%$ & 0,485 & 15,0 \\
\cline { 2 - 5 } & $480 \rightarrow 200 \mathrm{~W}$ & $9,17 \pm 0,17 \%$ & 0,449 & 35,0 \\
\hline & $280 \rightarrow 200 \mathrm{~W}$ & $7,19 \pm 0,09 \%$ & 0,388 & 63,5 \\
\hline
\end{tabular}

\subsection{Teores de Compostos Bioativos}

Os resultados obtidos para os teores de compostos bioativos na secagem contínua e intermitente por micro-ondas são apresentados na Figura 2. Verificou-se uma alta degradação dos mesmos se comparados aos teores presentes na Spirulina in natura. Isso mostra que, apesar do tempo reduzido de secagem, o material apresentou-se sensível à exposição a ondas eletromagnéticas como as do micro-ondas, gerando, portanto, uma redução dos compostos da microalga.

Em relação ao Teor de Fenólicos Totais (TPC), houve uma redução de 35\% em relação à Spirulina in natura na secagem contínua. Já na secagem intermitente, esse teor apresentouse $80 \%$ maior que o obtido em secagem contínua, sendo mais eficiente nas combinações de potências $600 \mathrm{~W} \rightarrow 280 \mathrm{~W}$ e $480 \mathrm{~W} \rightarrow 280 \mathrm{~W}$. Além disso, o tempo de secagem foi inferior, apresentando-se como uma combinação entre os tempos obtidos em cada potência na forma contínua (Figura 2a).

Em relação ao Teor de Flavonoides Totais (TFC), a redução dos teores no método de secagem contínua foi ainda maior que a redução no TPC: cerca de $42 \%$. Já na secagem intermitente foram obtidos resultados melhores, principalmente para a combinação de 


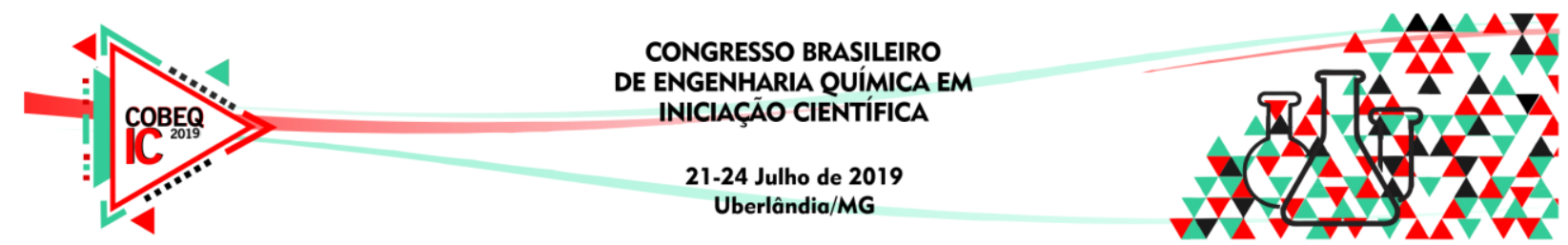

potências $800 \mathrm{~W} \rightarrow 280 \mathrm{~W}$, na qual foi observado um aumento de $30 \%$ da combinação em relação ao uso dessas potências na forma contínua (Figura $2 b$ ).

Em relação à quantidade de ácido cítrico presente na amostra (Acidez - ATT), houve também uma redução em seus teores, sendo esta de cerca de $22 \%$ em secagem contínua. Já em secagem intermitente, os resultados obtidos foram bem semelhantes aos obtidos na secagem contínua, indicando que o uso da intermitência em específico para esse composto não foi tão eficiente como para os fenólicos e flavonoides (Figura 2c).

Figura 2: Comparação dos resultados obtidos para os teores de compostos bioativos na secagem intermitente da Spirulina por micro-ondas: (a) TPC; (b) TFC; (c) ATT e (d) TF.
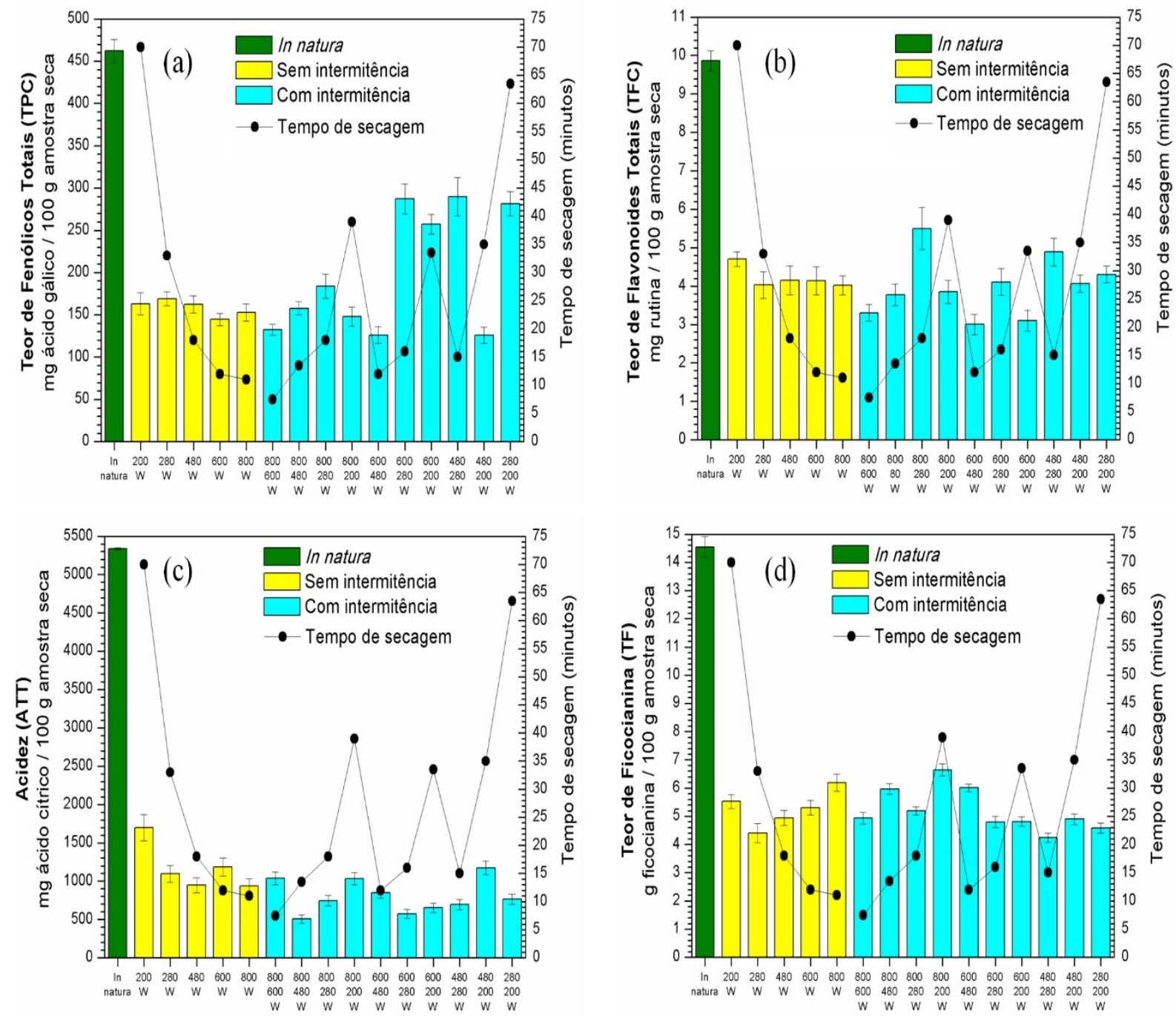

Em relação ao Teor de Ficocianina (TF) (Figura 2d) foi observada também uma redução de seus níveis em relação à amostra in natura, tanto em secagem contínua quanto em intermitente. No entanto, essa redução é menor quando utilizam-se potências mais altas em secagem contínua, como por exemplo $800 \mathrm{~W}$, sendo esta sua potência ideal de secagem. 


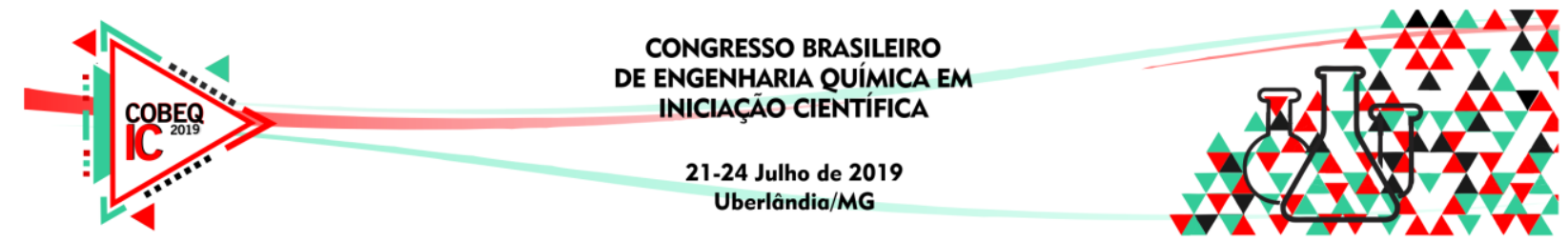

Apesar de tal constatação, ainda assim o TF foi reduzido em aproximadamente $57 \%$ em relação aos valores iniciais. Já em secagem intermitente, os resultados obtidos não foram muito significativos, sendo bem semelhantes aos obtidos em secagem contínua, assim como ocorreu nos teores de ácido cítrico.

\section{CONCLUSÃO}

A partir desses resultados, pôde-se concluir que a secagem em micro-ondas foi um método eficiente para a remoção de umidade da microalga Spirulina platensis, principalmente se for realizada de forma intermitente, a qual apresenta ainda mais vantagens se comparada à secagem de forma contínua. Em relação ao TPC, obtiveram-se condições melhores em potências de $600 \mathrm{~W} \rightarrow 280 \mathrm{~W}$ e $480 \mathrm{~W} \rightarrow 280 \mathrm{~W}$; quanto ao TFC, a melhor combinação de potências obtida foi de $800 \mathrm{~W} \rightarrow 280 \mathrm{~W}$. Já em relação ao ATT e FC, os resultados obtidos em ambos os métodos de secagem foram semelhantes no que tange à conservação desses compostos no material. Apesar de apresentar degradação nos teores de compostos bioativos, ainda assim foi possível obter, por esse método, condições específicas de potência para sua secagem e posterior utilização da microalga.

\section{REFERÊNCIAS}

AOAC. Official methods of analysis. Association of Official Analytical Chemists, Gaithersburg, MD, 1995.

COSTA, B. R.; RODRIGUES, M. C. K.; ROCHA, S. F.; POHNDORF, R. S.; LARROSA, A. P. Q.; PINTO, L. A. A. Optimization of Spirulina sp. Drying in heat pump: Effects on the physicochemical properties and color parameters. J. Food Process. Pres., v. 40, p. 934-942, 2016.

DESMORIEUX, H.; HERNANDEZ, F. Biochemical and Physical Criteria of Spirulina After Different Drying Processes. Drying 2004 - Proceedings of the 14th International Drying Symposium (IDS 2004), São Paulo-Brasil, vol. B, p. 900-907, 2004.

DOKE JR., J. M. An Improved and Efficient Method for the Extraction of Phycocyanin from Spirulina sp., Int. J. Food Eng., v. 1(5), p. 1-13, 2005.

SINGLETON, V. L.; ROSSI, J. A. Colorimetry of total phenolics with phosphomolibidic phosphotungistic acid reagents. Am. J. Enol. Viticult, v. 16, p. 144-158, 1965.

VENKATESH, M. S.; RAGHAVAN, G. S. V. An Overview of Microwave Processing and Dielectric Properties of Agrifood Materials, Biosyst. Eng., v. 88(1), p. 1-18, 2004.

ZHISHEN, J.; MENGCHENG, T.; JIANMING, W. The determination of flavonoid contents in mulberry and their scavenging effects on superoxide radicals. Food Chem., v. 64, p. 555-559, 1999. 